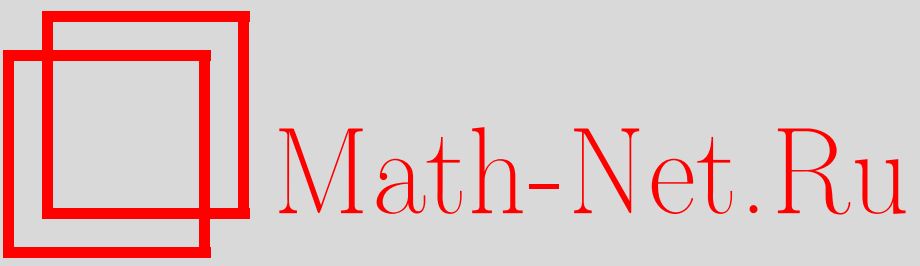

Д. Луккассен, Об оценках эффективной энергии для усредненного уравнения Пуассона с $p$-лапласианом, УМH, 1996, том 51, выпуск 4, 161-162

DOI: https://doi.org/10.4213/rm1004

Использование Общероссийского математического портала Math-Net.Ru подразумевает, что вы прочитали и согласны с пользовательским соглашением

http://www.mathnet.ru/rus/agreement

Параметры загрузки:

IP: 54.89 .56 .158

26 апреля 2023 г., 14:31:41 


\section{ОБ ОЦЕНКАХ ЭФФЕКТИВНОЙ ЭНЕРГИИ ДЛЯ УСРЕДНЕННОГО УРАВНЕНИЯ ПУАССОНА С $p$-ЛАПЛАСИАНОМ}

\section{Д. ЛУККАССЕН}

В этой работе мы получаем оценки эффективной энергии усредненной задачи для уравнения Пуассона с $p$-лапласианом. Мы доказываем, что полученное неравенство является точным и даже находим все случаи, когда верхняя и нижняя границы совпадают.

$\mathrm{B} \mathbb{R}^{N}$ мы рассматриваем параллелепипед $Y=\prod_{j=1}^{N}\left(0, x_{j}^{0}\right)(Y-$ ячейка). Мы изучаем семейство уравнений Пуассона с $p$-лапласианом

$$
-\operatorname{div}\left(\lambda\left(\frac{x}{\varepsilon}\right)|D u|^{p-2} D u\right)=f \text { в } \Omega \subset \mathbb{R}^{N}, \quad p>1,
$$

с краевыми данными Дирихле, где $\lambda-Y$-периодическая, ограниченая сверху и снизу положительными константами, функция, $\varepsilon$ - мальй параметр. Используя результаты усреднения монотонных операторов (см. [3]), мы получаем усредненную задачу в форме

$$
-\operatorname{div}(b(D u))=f \quad \text { в } \Omega,
$$

где усредненньй оператор $b: \mathbb{R}^{N} \rightarrow \mathbb{R}^{N}$ принимает следующий вид:

$$
b(\xi)=\frac{1}{|Y|} \int_{Y} \lambda(x)\left|\xi+D w^{\xi}(x)\right|^{p-2}\left(\xi+D w^{\xi}(x)\right) d x
$$

где $w^{\xi}$ - единственное (с точностью до постоянного слагаемого) решение локальной задачи:

Найти $w^{\xi} \in W_{\text {per }}^{1, p}(Y)$ (= пространство всех $Y$-периодических функций $\left.\psi \in W_{\text {loc }}^{1, p}\left(\mathbb{R}^{N}\right)\right)$ такую, что

$$
\int_{Y}\left(\lambda(x)\left|\xi+D w^{\xi}(x)\right|^{p-2}\left(\xi+D w^{\xi}(x)\right), D v\right) d x=0
$$

для всех функций $v \in W_{\text {per }}^{1, p}(Y)$.

Можно доказать (см. [1] и имеющиеся там ссылки), что $w^{\xi}$ реализует минимум в вариационной задаче:

$$
f_{\lambda}(\xi)=\min _{u \in W_{\text {per }}^{1, p}(Y)} \frac{1}{|Y|} \int_{Y} \lambda(x)|\xi+D u(x)|^{p} d x
$$

Заметим также, что, подставляя $v=w^{\xi}$ в (1), мы получим:

$$
(b(\xi), \xi)=f_{\lambda}(\xi)
$$

В этой работе исследуются неравенства для эффективной энергии $f_{\lambda}=(b(\cdot), \cdot)$, которые дают ее двусторонние оценки через некоторые интегральные средние от функции $\lambda$ (см. [4, с. 148]). Эти неравенства обобщают оценки Винера на уравнение Пуассона с $p$-лапласианом. В частности, при $p=2$ (т.е. для линейной проводимости) рассматриваемые неравенства совпадают с оценками, упомянутыми в [5]. В ряде случаев определяемые этими неравенствами нижние и верхние границы для эффективной энергии достаточно близки друг к другу и дают для нее лучшие оценки, чем полученные Хашином и Штрикманом. Ранее отмечалось [6], что в некоторых случаях получаемые нижняя и верхняя границы совпадают, вследствие чего эфффективная энергия вычисляется точно. В этой работе описываются все такие случаи. 
Если $s \in \mathbb{R}^{1}$ и $t \in \mathbb{R}^{N-1}$, то $x=(s, t)$ обозначает точку из $\mathbb{R}^{N}$ с координатами $x_{i}=s$, $x_{j}=t_{j}$ при $j<i$ и $x_{j}=t_{j-1}$ при $i<j$. Пусть $T=\prod_{j \neq i}\left(0, x_{j}^{0}\right)$ и $S=\left(0, x_{i}^{0}\right)$ с лебеговыми мерами $\eta$ и $\mu$, соответственно. Обозначим

$$
q_{i}=|Y|^{-1} \int_{Y} \lambda_{i} d x, \quad q^{i}=\left(|Y|^{-1} \int_{Y}\left(\lambda^{i}\right)^{1 /(1-p)} d x\right)^{1-p},
$$

где $\lambda_{i}(s, t)=\left(\frac{1}{|S|} \int_{S}(\lambda(\cdot, t))^{\frac{1}{1-p}} d \mu\right)^{1-p}$ для всех $s \in S$ и почти всех $t \in T$, а $\lambda^{i}(s, t)=$ $\frac{1}{|T|} \int_{T} \lambda(s, \cdot) d \eta$ для почти всех $s \in S$ и всех $t \in T$.

Лемма 1. Предположим, что $f \in L^{p}(Y)$, где $p \geqslant 1$, и пусть $U_{1}$ и $U_{2}-$ подмножества $Y$ вида $I_{i 1} \times \prod_{j \neq i} I_{j}$ и $I_{i 2} \times \prod_{j \neq i} I_{j}$, соответственно, где $\prod_{j \neq i} I_{j} \subseteq T,\left\{I_{j}\right\}$ отрезки, и $I_{i 1}, I_{i 2}$ - непересекающиеся отрезки $S$. Более того, предположим, что для всех таких пар $\left(U_{1}, U_{2}\right)$

$$
\left|U_{1}\right| \int_{U_{2}} f d x=\left|U_{2}\right| \int_{U_{1}} f d x .
$$

Тогда $f$ не зависит от $i$-й координатьи.

Теорема 1. Следующие утверждения эквивалентны: (а) $q_{i}=\left(b\left(e_{i}\right), e_{i}\right)$, (b) $\left(b\left(e_{i}\right), e_{i}\right)=q^{i}$, (c) $\lambda=k_{i 1} k_{i 2}$, әде $k_{i 1}$ зависит только от $i$-й координатьи, а $k_{i 2}$ не зависит от $i$-й координаты.

СлЕДСТВИЕ 1. $q_{i}=q^{i}$ для $i=1,2, \ldots, N$, если и только если $\lambda=\lambda_{1} \cdot \lambda_{2} \cdot \ldots \cdot \lambda_{N}$, əде $\lambda_{j}-$ функция только $j$-й координатьи.

ДокАЗАТЕЛЬСтво. Из теоремы 1 мы получаем следующие $N-2$ уравнения:

$$
\begin{aligned}
& k_{11}(0) k_{12}\left(x_{2}, x_{3}, \ldots, x_{N-1}, x_{N}\right)=k_{21}\left(x_{2}\right) k_{22}\left(0, x_{3}, x_{4}, \ldots, x_{N-1}, x_{N}\right) \text {, } \\
& k_{21}(0) k_{22}\left(0, x_{3}, \ldots, x_{N-1}, x_{N}\right)=k_{31}\left(x_{3}\right) k_{32}\left(0,0, x_{4}, \ldots, x_{N-1}, x_{N}\right) \text {, } \\
& k_{(N-2) 1}(0) k_{(N-2) 2}\left(0,0, \ldots, 0, x_{N-1}, x_{N}\right)=k_{(N-1) 1}\left(x_{(N-1)}\right) k_{(N-1) 2}\left(0,0, \ldots, 0, x_{N}\right) .
\end{aligned}
$$

Отсюда легко вывести, что $\lambda=\lambda_{1} \lambda_{2} \cdots \lambda_{N}$, где $\lambda_{j}$ зависит только от $j$-й координаты. Доказательство закончено.

\section{СПИСОК ЛИТЕРАТУРЫ}

[1] Chiado Piat V., Dal Maso G., Defranceschi A. // Ann. Inst. H. Poincaré. Anal. Non Linéaire. 1990. V. 7. P. 123-160. [2] Dal Maso G. An introduction to $\Gamma$-convergence. Boston: Birkhäuser, 1993. [3] Defranceschi A. An introduction to homogenization and $G$-convergence // Lecture notes, School on homogenization, ICTP, Trieste, September 6-17, 1993. [4] Харди Г.Г., Литтльвуд Д.Е., Полиа Г. Неравенства. М.: ИЛ, 1948. [5] Луккассен Д. // УМН. 1994.. Т. 49. № 4. C. 115. [6] Lukkassen D., Persson L.E., Wall P. On some sharp bounds for the homogenized $p$-Poisson equation // Research Report, Dept. of Math., Luleå University (to appear).

Норвегия, Нарвик

Технологический институт

Кафедра математики
Принято редколлегией 21.06.1996 